\title{
Recognition of lung volume condition based on phase space mapping using electrical impedance tomography
}

\author{
Mohammad Karimi Moridani ${ }^{1,2}$, Fatemeh Choopani ${ }^{1}$ and Mandana Kia ${ }^{1}$ \\ 1. Department of Biomedical Engineering, Faculty of Health, Tehran Medical Sciences, Islamic Azad University, Tehran, Iran \\ 2. E-mail any correspondence to: karimi.m@iautmu.ac.ir
}

\begin{abstract}
The purpose of this paper is to identify differences between abnormal and normal lung signals gathered by an EIT device, which is a new, non-invasive system that seeks the electrical conductivity and permittivity inside a body. Lung performances in patients are investigated using Phase Space Mapping technique on Electrical EIT signals. The database used in this paper contains 82 registered records of 52 individuals with proper lung volume. The results of this paper show that as the delay parameter $(\tau)$ increases, the SD1 parameter of phase space mapping indicates a significant difference between normal and abnormal lung volumes. The value of the SD1 parameter with $\tau=6$ in the case that the lung volume is in a normal condition is $342.57 \pm 32.75$ while it is $156.71 \pm 26.01$ in nonoptimal mode. This method can be used to identify the patients' lung volumes with chronic respiratory illnesses and is an accurate assessment of the diverse methods to treat respiratory system illnesses in addition to saving various therapeutic costs and dangerous consequences that are likely to occur by using improper treatment methods. It can also reduce the required treatment durations.
\end{abstract}

Keywords: lung volume; electrical impedance tomography; signal processing; phase space mapping

\section{Introduction}

A great number of people die annually due to various kinds of lung diseases, which can cause impariment and respiratory disorders that are mostly caused by air pollution, tobacco consumption, genetic tribulation, etc. Lungs are the organs of the body which expand and contract hundreds of times durung the day separating carbon dioxide from oxygen. Their disorders can cause serious problems in the whole respiratory system [1]. These pulmonary diseases are namely: Asthma, chronic obstructive pulmonary diseases (COPD), pneumonia, tuberculosis, lung cancer, etc. Asthma is an illness which can be detected by cough and origins from the inflammation of the respiratory tract. It is mostly caused by external factors such as dust, the breath of animals or some specific plants. This illness appears by the reaction of the body's immune system $[2,3]$.

Chronic obstructive pulmonary diseases (COPD) consists of bronchitis and emphysema. Chronic bronchitis create sputum, however, emphysema may cause other kinds of lung disorders. The majority of patients usually suffer from both [4]. Chronic bronchitis is created by a mucus overproduction of the lung cells, and it declines the lung function, which increases the risk of airway obstructions, raises the risk of respiratory infection and could even cause death [5].

Pneumonia is an infectious diseases among adults in which the bacteria enters to the body from the individual's respiratory tracts [6].

Tuberculosis is another bacterial and also contagious disease which spreads through blood or lymph node [7].

Furthermore, lung cancer is an illness which is contained from myriad forms. The most prevalent of those takes place in the center of the lung lobes [8].

Electrical Impedance Tomography (EIT) is a non-invasive imaging modality which possesess a lot of potential in industry and the medical field. This method applies electrical stimulation and related measurements at the body surface to extract the electrical characteristics of internal tissues. 
The distribution of electrical conductivity within a tissue could be captured by applying an electrical current to the tissue and measuring the surface's voltage divisions [9].

Furthermore, EIT can be applied bedside as a continuous monitoring technique, as it only requires small devices and does not expose patients or users to any ionized radiation. The EIT technique was introduced in the early 1980s by Barber and Brown. Soon after, a broad spectrum of possible applications in medicine was suggested, ranging from gastric emptying to brain function monitoring and from breast imaging to lung function assessment. This method was tested on acute respiratory distress syndrome by measuring surface voltages frequently using electrodes [10-11].

In electrical impedance tomography, current is injected from electrodes placed arround the chest, then the resulting surface voltages are measured [12]. In 1985, clinical diagnostic software was provided using EIT, which is a convenient method for lung imaging [13]. In 1999, by using the EIT method it was concluded that the electrical properties for each section of a tissue is different from the others [14]. According to research by Victorino and his colleagues, measured imbalance in air ventilation in each different region of the lungs was due to pulmonary damages. By comparing EIT and CT methods, the EIT was introduced as a great procedure for diagnosis of lungs disorders [15]. EIT images were identified as well as CT images and as a result, EIT had the advantages of both being non-invasive and free of radiation. [17-18]. In 2016, research was done on a number of anesthetized rats and EIT images of cortical neural activities were obtained [19]. Additionlly, some studies were assessed in the fields of the various patterns of the EIT measurement to maximize the heart rate signal in parallel with lungs by Tobias Menden and his colleague in 2017. They found that the heart rate signal was completely different from the respiration signal [20]. In 2017 Chitturi and his colleague focused on important techniques to upgrade EIT's spatial system [21]. Hanan and her colleagues have performed another research and found out an essential tool for understanding neural circuits by using EIT [22].

We used EIT signal as a non-invasive method for evaluating lung volumes and comparing the differences between normal and abnormal lungs with mathematic techniques such as Phase Space methods.

\section{Materials and methods}

\section{Data base}

Data used in this paper includes 82 registered signals recorded from 52 patients with lung disorders collected by Dr. Hashemian from the Masih Daneshvari Hospital of Tehran. The sampling frequency is $20 \mathrm{~Hz}$ and data are mostely recorded from patients who have had a surgery and were using artificial respiration. For recording the data, a belt with 16 electrodes attached to the device was utilized. This belt have got the ability of adjustment according to the size of an adult's body and closes below the patient's breast, right around the lung. The outputs consist of electrical impedance signals of four regions of lungs according to fig. 1 . These regions are numbered from 1 to 4 . A sample of registrated signals from each region using the EIT system, is depicted in fig. 2. The total of four region's signals (ROI1 to ROI4) make up the Global signal.

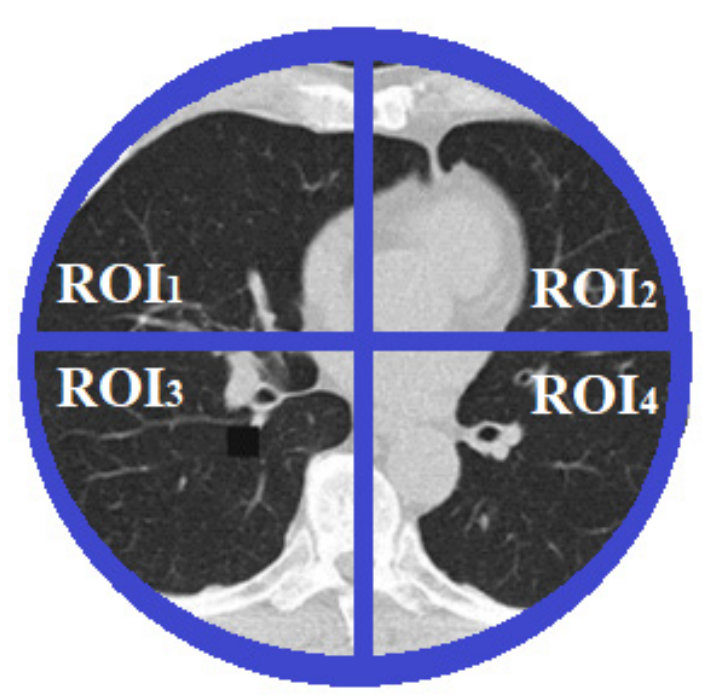

Fig. 1. Lungs Classification to four regions.

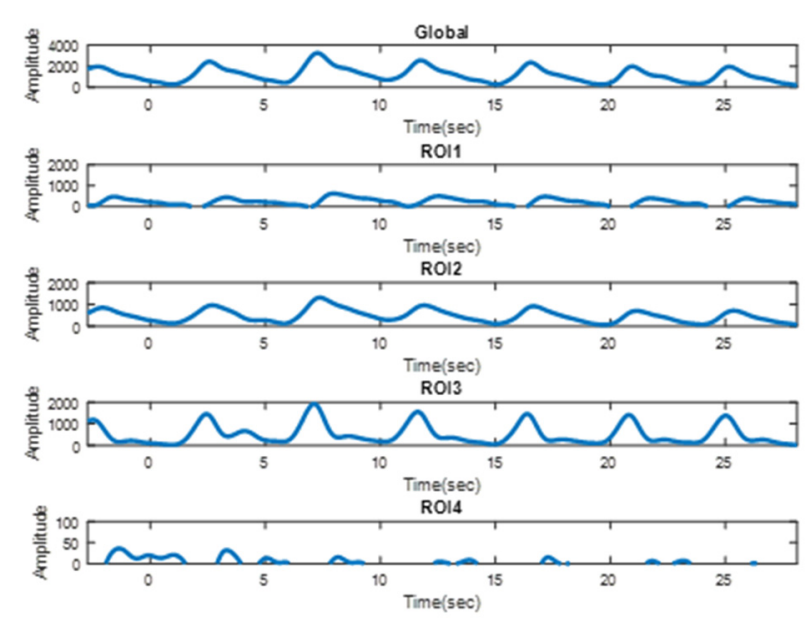

Fig. 2. Electrical Impedance Tomography of lung's four regions.

\section{Suggested method}

In this paper, the state of lung volume after surgery has been considered before non-linear signal processing. Comparing the results can make a significant contribution for doctors and aiding them to a better detection of lungs function. Looking further, since EIT signals with time and space accuracy cannot be detected by lung's volume which was obtained before and after surgery, a better recognition of lung volume condition named Phase Space Method was used to introduce an indicator for comparing the states of the lung's volume throughout and after the surgery. 


\section{Phase space}

Recent studies about medical signal processing illustrate that due to the nature of the non-linearity of the EIT signal, linear methods do not succeed in extracting useful information from the signal and cannot classify with high accuracy between normal signals and patient's signals. Thus, it can be observed that today, in various studies the phase space method is used to diagnose among normal signals and patient signals [23-25].

Assuming that the input signal of $s(n)=s 1, s 2, \ldots$, sn has been defined for making phase space, the $s(n)$ signal was divided into two signals called $x 1$ and $x 2$. In this formula $\tau$ is the delay parameter.

$$
\begin{gathered}
x_{1}: s_{2} \cdot s_{3} \ldots s_{n} \\
x_{2}: s_{1} \cdot s_{2} \ldots s_{n-\tau}
\end{gathered}
$$

Next, for creating the phase space, $\mathrm{x} 1, \mathrm{x} 2$ and $\tau=1$ are drawn in accordance with each other. $\tau=1$ means that every sample is possessed in accordance with the next sample of itself. For making a distinction, different phase spaces must be introduced to the features of this mapping. In this paper, deviation criteria for short-term and long-term changes are namely: SD1 (Standard Deviation) and SD2 which are the features of this mapping. SD1 and SD2 are dispersions of points perpendicular to the axis of line-of-identity. Formulas (2) show how to calculate SD1 and SD2:

$$
\begin{gathered}
S D_{1}=\frac{\sqrt{2}}{2} \operatorname{std}\left(x_{1}-x_{2}\right) \\
S D_{2}=\sqrt{2 \operatorname{std}\left(x_{1}\right)^{2}-0.5 \operatorname{std}\left(x_{1}-x_{2}\right)^{2}}
\end{gathered}
$$

Figure 3 shows the phase space for one EIT signal sample and parameters introduced in this mapping.

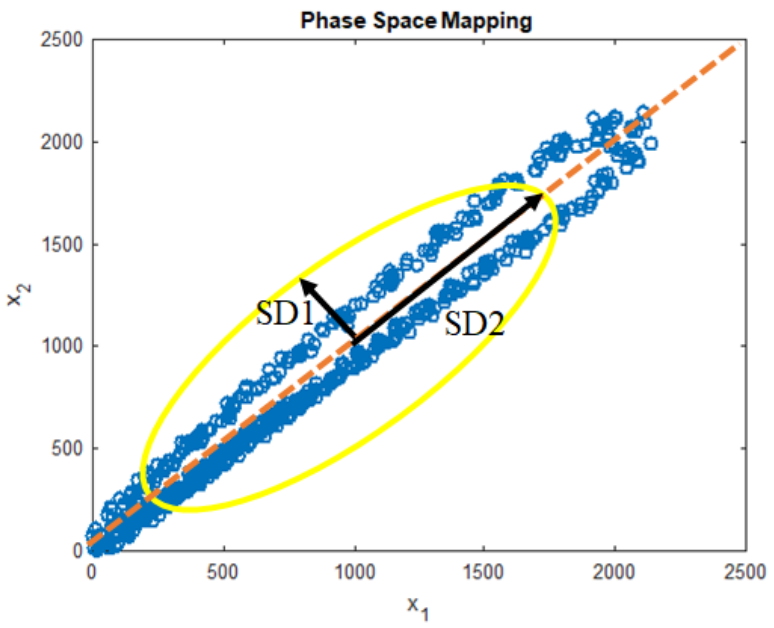

Fig. 3. Phase space mapping.

\section{Informed consent}

Informed consent has been obtained from all individuals included in this study.

\section{Ethical approval}

The research related to human use has been complied with all relevant national regulations, institutional policies and in accordance with the tenets of the Helsinki Declaration, and has been approved by the authors' institutional review board or equivalent committee.

\section{Results}

As mentioned before, the temporal data of volume signals cannot be well differentiated by the arrival of lung volume to the desired state. In this paper, the idea of applying the nonlinear method and converting the lung volume signal into phase space maps was presented. Figures 4 and 5 delineate the phase space mapping of a suitable and an unsuitable Global Signal respectively. As it can be observed, no information can be extracted to understand the condition of lungs' volume by visual comparison of Figs. 3 and 4 .

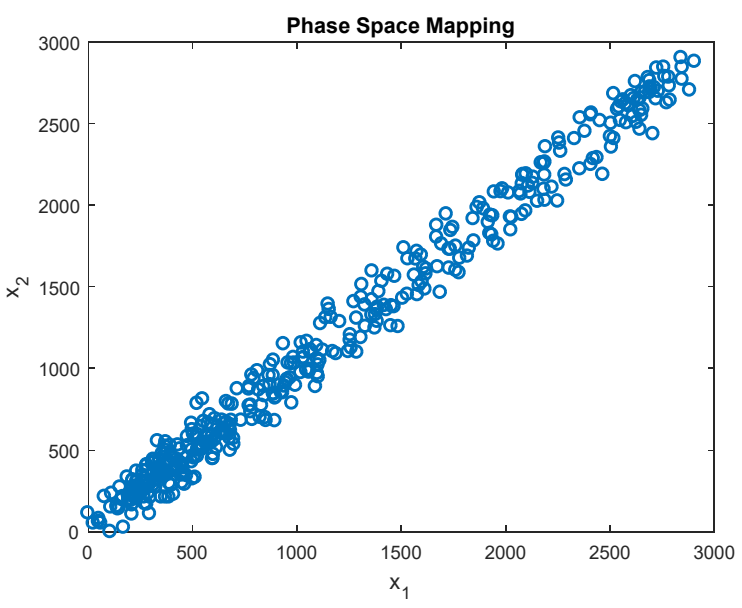

Fig. 4. A phase space mapping for suitable lung volume.

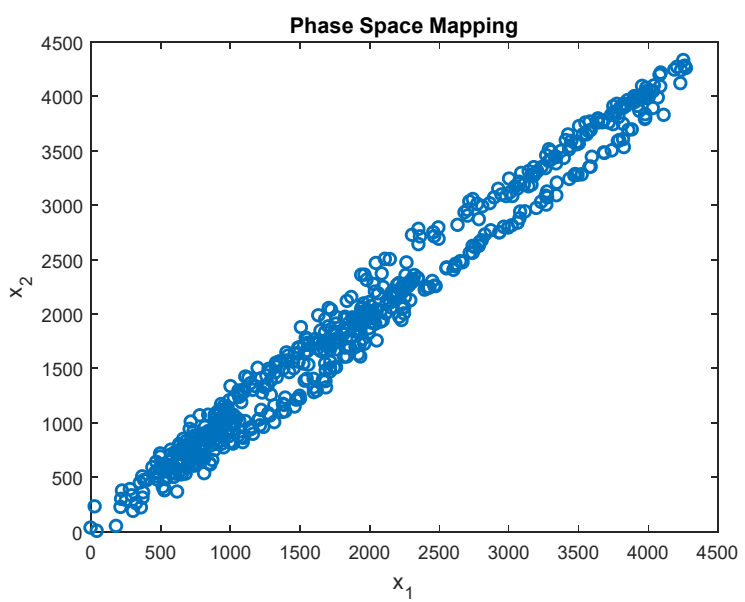

Fig. 5. A phase space mapping for unsuitable lung volume.

Furthermore, the calculation of SD1 and SD2 parameters of two modes (suitable and unsuitable lung volume) have also showed similar results. Table 1 presents the average results and the standard deviation of the SD1 and SD2 features in the case which lung volume is present in two appropriate and inappropriate situations. The statistical analysis were used to distinguish between two 
groups (before and after surgery). And as a result, the value of destination between the two groups with $P$ - value was considered less than 0.05 . All the analyses were obtained using MATLAB 2018.

\section{Implementation of the delay parameter in phase space mapping}

The Phase Space Mapping introduced in the previous section is plotted with the delay parameter value $(\tau)$, equal to 1 . For a better discrimination between two groups, Phase Space Mapping was reconstructed by applying different delay parameters. Values in Table 2 indicate the mean and standard deviations of the SD1 and SD2 criterion for the delay parameter of 2 to 6 . The statistical comparison of the results denotes the meaningful distinction of the SD1 property with increasment of $\tau$. So that when the volume is abnormal and the amount of $\tau$ is considered 6 , the average value of SD1 is twice that of the normal case ( $P$-value $<0.05)$. This means that the EIT signal in the case that a person's lung is in a suitable condition shows a higher uniformity in the four regions.

TABLE 1: The average and the standard deviation of the $\mathrm{SD}_{1}$ and $\mathrm{SD}_{2}$ parameters with $\tau=1$.

\begin{tabular}{|c|c|c|}
\hline \multirow{2}{*}{$\begin{array}{c}\text { Lung Volume } \\
\text { Conditions }\end{array}$} & SD1 & SD2 \\
\cline { 2 - 3 } & Mean \pm Standard deviation & Mean \pm Standard deviation \\
\hline Unacceptable & $89.23 \pm 10.45$ & $1914.71 \pm 203.61$ \\
\hline Suitable & $94.43 \pm 11.76$ & $11673.52 \pm 174.11$ \\
\hline
\end{tabular}

TABLE 2: The average and the standard deviation of the $\mathrm{SD}_{1}$ and $\mathrm{SD}_{2}$ parameters with $\tau=2$.

\begin{tabular}{|c|c|c|}
\hline \multirow{2}{*}{$\begin{array}{c}\text { Lung Volume } \\
\text { Conditions }\end{array}$} & SD1 & SD2 \\
\cline { 2 - 3 } & Mean \pm Standard deviation & Mean \pm Standard deviation \\
\hline Unacceptable & $96.51 \pm 12.10$ & $2104.23 \pm 223.25$ \\
\hline Suitable & $112.67 \pm 24.09$ & $1716.06 \pm 213.43$ \\
\hline
\end{tabular}

TABLE 3: The average and the standard deviation of the $\mathrm{SD}_{1}$ and $\mathrm{SD}_{2}$ parameters with $\tau=3$.

\begin{tabular}{|c|c|c|}
\hline \multirow{2}{*}{$\begin{array}{c}\text { Lung Volume } \\
\text { Conditions }\end{array}$} & SD1 & SD2 \\
\cline { 2 - 3 } & Mean \pm Standard deviation & Mean \pm Standard deviation \\
\hline Unacceptable & $118.19 \pm 15.75$ & $2217.25 \pm 228.51$ \\
\hline Suitable & $158.09 \pm 34.12$ & $1835.12 \pm 216.41$ \\
\hline
\end{tabular}

TABLE 4: The average and the standard deviation of the $\mathrm{SD}_{1}$ and $\mathrm{SD}_{2}$ parameters with $\tau=4$.

\begin{tabular}{|c|c|c|}
\hline \multirow{2}{*}{$\begin{array}{c}\text { Lung Volume } \\
\text { Conditions }\end{array}$} & SD1 & SD2 \\
\cline { 2 - 3 } & Mean \pm Standard deviation & Mean \pm Standard deviation \\
\hline Unacceptable & $132.54 \pm 21 / 14$ & $2375.78 \pm 234.01$ \\
\hline Suitable & $216.37 \pm 41 / 62$ & $1932.64 \pm 220.17$ \\
\hline
\end{tabular}

TABLE 5: The average and the standard deviation of the $\mathrm{SD}_{1}$ and $\mathrm{SD}_{2}$ parameters with $\tau=5$.

\begin{tabular}{|c|c|c|} 
parameters with $\tau=5$. & SD1 & SD2 \\
\hline $\begin{array}{c}\text { Lung Volume } \\
\text { Conditions }\end{array}$ & Mean \pm Standard deviation & Mean \pm Standard deviation \\
\cline { 2 - 3 } Unacceptable & $148.20 \pm 24.17$ & $2418.23 \pm 239.67$ \\
\hline Suitable & $297.41 \pm 49.25$ & $2098.27 \pm 226.15$ \\
\hline
\end{tabular}

TABLE 6 The average and the standard deviation of the $\mathrm{SD}_{1}$ and $\mathrm{SD}_{2}$ parameters with $\tau=6$.

\begin{tabular}{|c|c|c|}
\hline \multirow{2}{*}{$\begin{array}{c}\text { Lung Volume } \\
\text { Conditions }\end{array}$} & SD1 & SD2 \\
\cline { 2 - 3 } & Mean \pm Standard deviation & Mean \pm Standard deviation \\
\hline Unacceptable & $156.71 \pm 26.01$ & $2457.98 \pm 245.54$ \\
\hline Suitable & $342.32 \pm 57.75$ & $2113.18 \pm 230.67$ \\
\hline
\end{tabular}

Figures 6 and 7 illustrate feature changes of the SD1 and SD2 with a leap in $\tau$. The comparison of the obtained results shows that by increasing the delay parameter $(\tau)$, the value of the SD1 parameter rises, and when the amount of $\tau$ remained stable at 6 , there is a significant shift between appropriate $(342.57 \pm 32.75)$ and inappropriate (156.71 \pm 26.01) lung volume status. It is to be noted that the SD2 parameter does not show significant changes in the two desirable and undesirable lung conditions as $\tau$ rises.

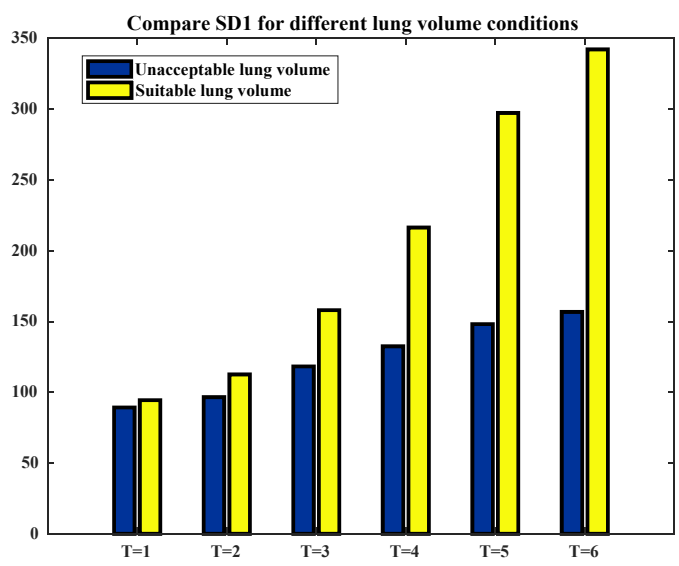

Fig 6. Comparison of the SD1 parameter for different value of the $\tau$.

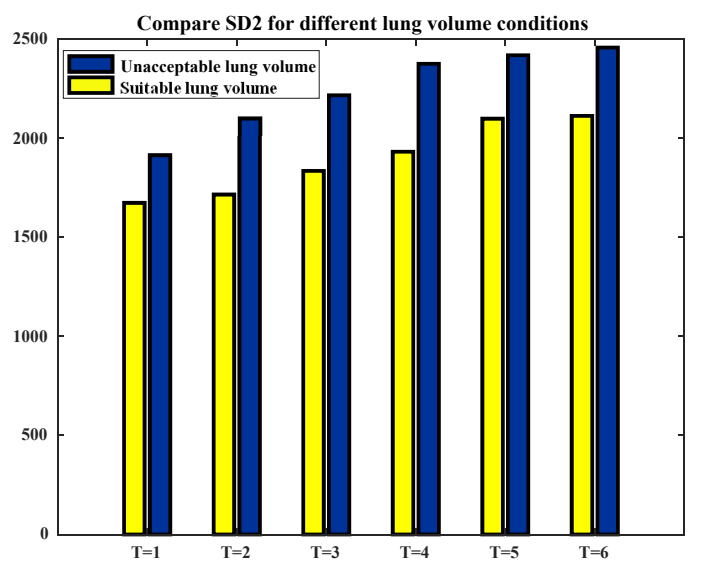

Fig. 7. Comparison of the SD2 parameter for different value of the $\tau$.

\section{Discussion}

In this paper, we use the method of Phase Space Mapping to compare the lung volume situations in different patients. With the help of the diagnostic computer system presented in this paper, a better understanding of the condition of the lung volume after surgery can be obtained. The results of the proposed method can provide valuable information about the function and the amount of patients' lung volumes for the information of doctors. In Gonzales's research in 2016, the use of $3 \mathrm{D}$ television systems was significantly more 
effective in the 3D EIT imaging than the Gaussian smoothing rule, which can be used in imaging of electrical tomography and optical diffusion tomography [9]. In a study in 2015, EIT, which controls lung's condition over time, is a significant step towards detection of lung diseases and can demonstrate patient conditions better than any other imaging method [10]. In 2012, Smith decided that in diseases like ARDS, patients are in serious need of artificial respiration with specific conditions (ECMO), for this very reason, it is highly significant for such patients' lungs' condition to be exhibited continuously in order to inform doctors about the changes that need to be applied in the ventilator device settings such as (HFOV) [11]. According to the data collected from the pulmonary investigation in 2000 , the smallest changes in the adsorbed oxygen are visible in detail [13]. In a paper from 1999, which was authored by Cheney and his colleagues, the mathematical model relations are defined for the EIT to be more accurate in some existing theories referred to by the adaptive current tomography (ACT), and after studies, an algorithm was selected that was the most effective one for making images [14].

In 2004, according to comparisons between dynamic computerized tomography in a heterogeneous population of critically ill patients under mechanical ventilation in different moments, Victorino and his colleagues concluded that, among variables derived from computerized tomography, changes in absolute air content was best explained by the integral of impedance changes inside the regions of interest [15]. Pelosi and his colleagues also carried out a study in which each lung was divided into 10 X-rays, and the hydrostatic pressure (SPL) was calculated separately, and the result was that the hydrostatic pressure in the lungs of ARDS patients is higher than the hydrostatic pressure in the lungs of healthy individuals [16]. In 2000, Newman and his colleagues tested some EIT experiments on 6 pigs to find similarities between them and their ARDS patients. It was concluded that in order to prevent lung collapse during artificial respiration, PEEP should be higher than $20 \mathrm{~cm}$ of water in less than 0.6 seconds while a PEEP with maximum of $15 \mathrm{~cm}$ of water in 1.4 seconds is necessary for these patients. Therefore, due to the differences in time, it cannot be stated that there are any similarities between patients and the pigs [17]. In another paper published in 2016, different tests have been done on brain systems of two mice in which, only one of them being injected in their brain to investigate the differences, and as a result, no changes were observed in any of them [18].

In 2017, Tobias and his colleagues compared the signals received from hearts and lungs, and the result was that the frequency spectrum of the heart had various zig-zag patterns, at lower measurement frequencies there were smaller patterns and vice versa [20]. In 2017, focusing on the previous research, the result was found that in a breast cancer case peak-to-peak distances represent disturbances of the breast, and Array Processing technique can be a step towards the development of spatial resolution for future EIT applications [21]. In 2018, Hanan and his colleagues have found two frequency responses in two impedance signals associated with epilepsy in mouse brain cortex, which is due to the ability of imaging the neural activity by this method of imaging as well as the metabolic changes [22].

Therefore, by applying the proposed method in this paper, we used informations about the individuals' lung volumes with chronic respiratory diseases and accurate assessmentss of the different methods to treat respiratory system disorders. This method can reduce the patient's desired treatment duration in addition to saving various therapeutic costs, which might be caused by using poor treatment methods that are also likely to give adverse consequences.

\section{Conflict of Interest}

The authors whose names are listed in this manuscript has no affiliation with or involvement in any organization or entity with any financial interest or nonfinancial interest in the subject matter or materials discussed in this paper. Authors have also declared that no conflict of interest exists.

\section{Acknowledgements}

We thank our colleague Afshin Mohammadkhani Motlagh for assistance with accessing required data and who provided insight and expertise that greatly assisted the research.

\section{References}

1. Kimura T, Kawakami T, Kikuchi A, Ooev R, Akiyama M, Horikoshi H. A Study on diagnostic assist systems of chronic obstructive pulmonary disease from medical images by deep learning. J Comput Comm. 2018;6,21-31. https://doi.org/10.4236/jcc.2018.61003.

2. Barnes KC. Genetic studies of the etiology of asthma. Proc Am Thorac Soc. 2011;8(2): 143-148. https://dx.doi.org/10.1513\%2Fpats.201103-030MS.

3. Kiley J, Smith R, Noel P. Asthma phenotypes. Curr Opin Pulm Med. 2007;13(1):19-23. https://doi.org/10.1097/MCP.0b013e328011b84b.

4. Anderson B, Brown H, Bruhl E, Bryant K, Burres H, Conner K, et al. Diagnosis and management of chronic obstructive pulmonary disease (COPD). Institute for Clinical Systems Improvement. 10th edition; 2017.

5. Kim V, Criner GJ. Chronic Bronchotis and chronic obstructive Pulmonary Disease. Am J Respir Crit Care Med. 2013;187(3): 228-237.

6. Richard T. Ellison III, Gerald R. Donowitz. Acute pneumonia. $8^{\text {th }}$ ed. Philadelphia, PA: J Microb, Immunol Infect. 2015.

7. Sekulich M. What is tuberculosis? Elsevier. Mark C, editor; 1994,104. https://doi.org/10.1016/C2013-0-06740-0. 
8. Chima A. Osuoha, Karen E. Callahan, Carmen P. Ponce, Paulo S. Pinheiro. Disparities in lung cancer survival and receipt of surgical treatment. Lung Cancer. 2018;122,54-5. https://doi.org/10.1016/j.lungcan.2018.05.022

9. Gonzalez G, Huttunen J, Kolehmainen V, Seppänen A, Vauhkonen, M. Experimental evaluation of 3D electrical impedance tomography with total variation prior. Inverse Problems in Science and Engineering. 2016;24(8):1411-1431. https://doi.org/10.1080/17415977.2015.1113961.

10. Teschner E, Imhoff M, Leonhardt S.Electrical Impedance Tomography: the realization of regional ventilation monitoring, 2nd edition; 2015.8-152.

11. Camporota L, Smith J, Barrett N, Beale R. Assessment of regional lung mechanics with electrical impedance tomography can determine the requirement for ECMO in patients with severe ARDS. Intensive Care Med. 2012;38(12):2086-7. https://doi.org/10.1007/s00134-012-2701-2.

12. Ross AS, Saulnier GJ, Newell JC, Isaacson D. Electrical Impedance Tomography. Journal of Physics: Conference Series 2001;18(6): 31-43. https://doi.org/10.1109/79.962276.

13. Frerichs I. Electrical Impedance Tomography (EIT) in applications related to lung and ventilation: a review of experimental and clinical activities, Physiol Meas.

2000;21(2):R1-21. https://doi.org/10.1088/0967-3334/21/2/201.

14. Cheney M, Isaacson D, Newell JC. Electrical Impedance Tomography. Society for Industrial and Applied Mathematics Philadelphia, PA, USA. SIAM Review 1999;41:85-101. https://doi.org/10.1137/S0036144598333613.

15. Victorino JA, Borges JB, Okamoto VN, Matos GF, Tucci MR, et al. Imbalances in regional lung ventilation: A validation study on electrical impedance tomography. Am J Respir Crit Care Med. 2004;169(7):791-800. https://doi.org/10.1164/rccm.200301-1330C.

16. Gattinoni L, D'Andrea L, Pelosi P, Vitale G, Pesenti A, Fumagalli R. Regional effects and mechanism of positive endexpiratory pressure in early adult respiratory distress syndrome. JAMA. 1993;269-2122-2127. https://doi.org/10.1001/jama.1993.03500160092039

17. Neumann P, Berglund JE, Andersson LG, Maripu E, Magnusson A, Hedenstierna G. Effects of inverse ratio ventilation and positive end expiratory pressure in oleic acidinduced lung injury. Am J Respir Crit Care Med. 2000; 161:1537-1545. https://doi.org/10.1164/ajrccm.161.5.9906060s

18. Neumann P, Berglund JE, Mondejar EF, Magnusson A, Hedenstierna G. Effect of different pressure levels on the dynamics of lung collapse and recruitment in oleic-acidinduced lung injury. Am J Respir Crit Care Med. 1998; 158:1636-1643. https://doi.org/10.1164/ajrccm.158.5.9711095

19. Aristovich KY, Packham BC, Koo H, Sato dos Santos G, McEvoy A, Holder DS. Imaging fast electrical activity in the brain with electrical impedance tomography. Neurolmage. 2016; 124:204-213. https://doi.org/10.1016/j.neuroimage.2015.08.071

20. Menden T, Orschulik J. Signal analysis of 2-belt EIT measurement patterns, $21^{\text {st }}$ International Student Conference on Electrical Engineering; 2017. Online: http://poseidon2.feld.cvut.cz/conf/poster/proceedings/ Poster_2017/Section_El/EI_036_Menden.pdf

21. Chitturi V, Nagi F. Spatial resolution in electrical impedance tomography: A topical review. J Electr Bioimp. 2017;66-78. http://dx.doi.org/10.5617/jeb.3350

22. Hannan S, Faulkner M, Aristovich K, Avery J, Walker M, Holder D. Imaging fast electrical activity in the brain during ictal epileptiform discharges with electrical impedance tomography. Neurolmage. 2018; 20:674-684. https://doi.org/10.1016/j.nicl.2018.09.004

23. Moridani MK, Setarehdan SK, Nasrabadi AM, Hajinasrollah E. New algorithm of mortality risk prediction for cardiovascular patients admitted in intensive care unit. Int J Clin Exp Med. 2015;8(6):8916-8926.

24. Moridani MK, Setarehdan SK, Nasrabadi AM, Hajinasrollah E. Non-linear feature extraction from HRV signal for mortality prediction of ICU cardiovascular patient. J Med Eng Technol. 2016;40(3):87-98. https://doi.org/10.3109/03091902.2016.1139201

25. Moridani MK, Kia M, Choopani F. Development of computeraided system to evaluate the lung efficiency using electrical impedance tomography. $3^{\text {rd }}$ International Conference on Electrical Engineering, Kharazmi University, Tehran, Iran; 2018. 